\title{
PLANIFICACIÓN FORESTAL EN ESPACIOS NATURALES PROTEGIDOS: HERRAMIENTAS INTEGRADORAS EN UN CONTEXTO DE CAMBIO
}

\author{
Míriam Piqué Nicolau \\ Área de Gestión Forestal Sostenible. Centre Tecnològic Forestal de Catalunya (CTFC). Crta. de Sant Llorenç \\ de Morunys, Km. 2. 25280-SOLSONA (Lleida, España).Correo electrónico: miriam.pique@ctfc.es
}

\section{Resumen}

Los espacios naturales protegidos (ENP) son aquellos dedicados a la protección de la diversidad biológica y de los recursos naturales y culturales asociados. Gran parte de estos ENP se encuentran distribuidos en una gran variedad de ecosistemas y formaciones forestales, y dentro de la Red Natura 2000. Sin embargo, en España, existe una gran disparidad de denominaciones para designar los ENP y una gran diversidad de normativas sectoriales y figuras de planificación a escala territorial que afectan a estos espacios, lo cual dificulta, a menudo, la implementación de figuras de planificación a nivel táctico y operativo y, en definitiva, la gestión final del ENP. La gestión forestal es clave para la conservación de gran parte de los ENP, con el objetivo de preservar la biodiversidad y los recursos naturales, en el marco de una gestión multifuncional y sostenible, que compatibilice la conservación con la producción y puesta en valor de los recursos naturales, e integre las necesidades del territorio y medidas de adaptación al cambio global. Para ello es necesaria la coordinación entre los distintos instrumentos de planificación del territorio. Las figuras de planificación a escala monte deben incorporar la zonificación y directrices de las figuras de planificación de escala espacial y jerárquica superior. Del mismo modo, las figuras de planificación de ámbito superior necesitan adaptarse a la realidad y necesidades que ponen de manifiesto las planificaciones a escalas menores.

Palabras clave: Conservación, Gestión forestal, Proyecto de ordenación forestal, Red Natura 2000, Cambio global, Criterios de conservación de la biodiversidad, Multifuncionalidad del monte, Valor del monte

\section{INTRODUCCIÓN}

Espacios Naturales Protegidos (ENP) en España

Según la Unión Internacional para la Conservación de la Naturaleza (UICN), un área protegida es "un espacio geográfico claramente definido, reconocido, dedicado y gestionado, mediante medios legales u otros tipos de medios eficaces, para conseguir la conservación a largo plazo de la naturaleza y de sus servicios ecosistémicos y valores culturales asociados" (DUDLEY, 2008).
En el ámbito español, la Ley LEY 42/2007, de 13 de diciembre, del Patrimonio Natural y de la Biodiversidad, establece la normativa básica sobre las áreas protegidas. De acuerdo a esta ley, los Espacios Naturales Protegidos (ENP) son aquellos que cumplen al menos uno de los requisitos siguientes:

- Contener sistemas o elementos naturales representativos, singulares, frágiles, amenazados o de especial interés ecológico, científico, paisajístico, geológico o educativo. 
- Estar dedicados especialmente a la protección y el mantenimiento de la diversidad biológica, de la geodiversidad y de los recursos naturales y culturales asociados.

En función de los bienes y valores a proteger y de los objetivos de gestión a cumplir, los ENP se clasifican en cinco categorías básicas de ámbito estatal (Parques Nacionales, Parques Naturales, Reservas Naturales, Monumentos Naturales, Paisajes Protegidos y Áreas Marinas Protegidas). Sin embargo, dado que la mayoría de las Comunidades Autónomas han ido desarrollando su propia normativa a lo largo de estos años, existen en la actualidad en España más de 40 denominaciones distintas para designar los ENP (MAGRAMA, 2013a).

Así pues, en España alrededor del $27 \%$ del territorio está bajo una figura de espacio protegido, siendo la de Parque Natural (Figuras 1 a 4) la que engloba más del $63 \%$ de la superficie declarada como ENP (EUROPARC ESPAÑA, 2013).

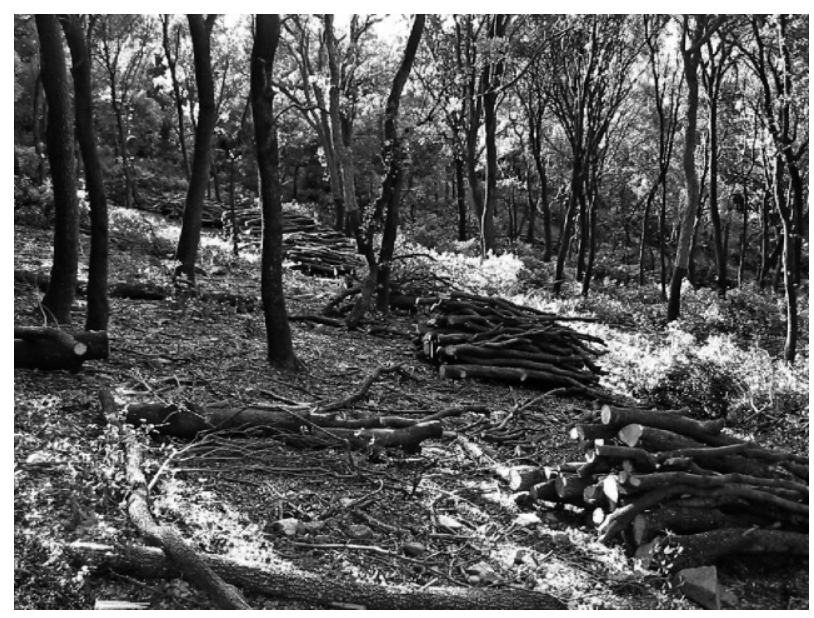

Figura 1. Parque Natural del Montseny (Girona). Aprovechamiento de leñas mediante gestión irregular en una masa mixta de encina y alcornoque. Autor: AGS-CTFC

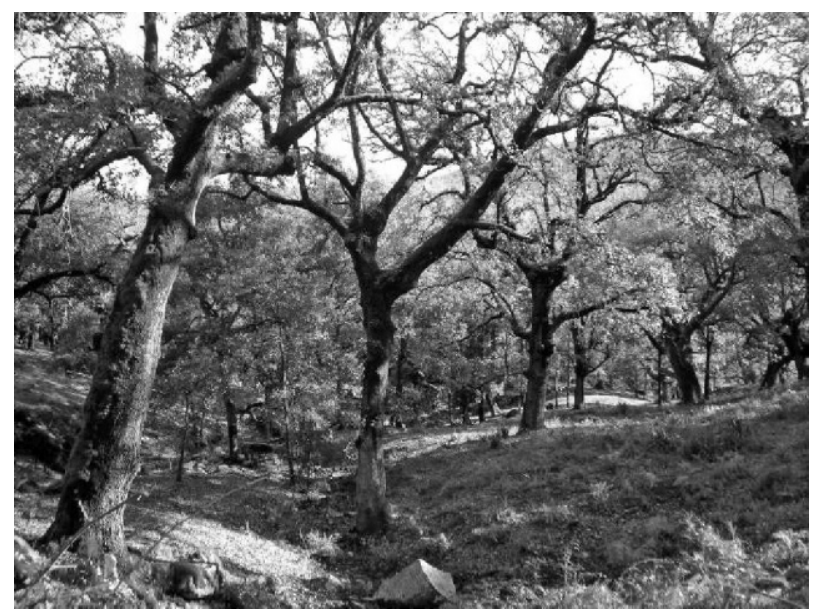

Figura 2. Parque Natural de los Alcornocales. Cortes de la Frontera (Málaga). Rodal de bosque maduro de Quercus canariensis. Autora: Raquel Saiz. 


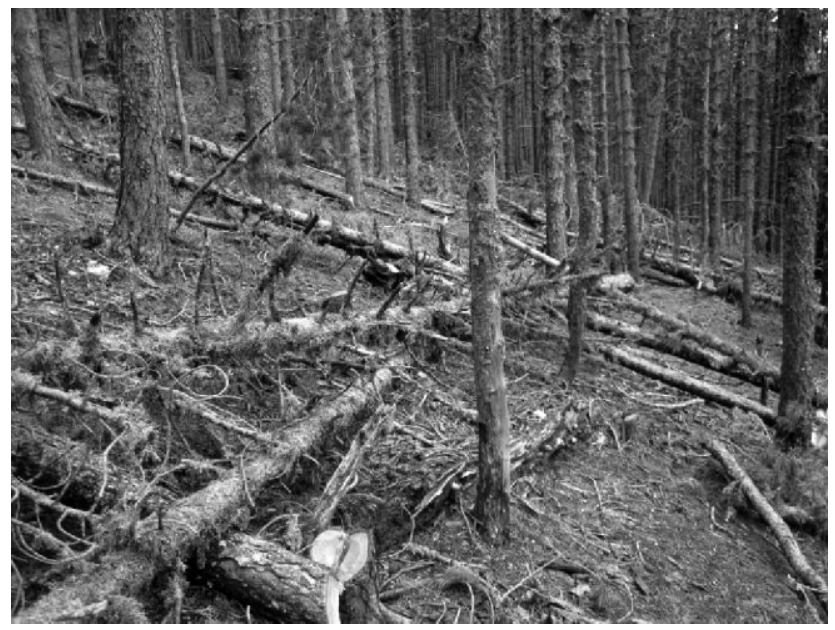

Figura 3. Parque Natural del Alt Pirineu (Lleida). Cortas de pequeños bosquetes en masa regular de Pinus uncinata con el objetivo de diversificar la estructura de la masa y mejorar el hábitat del urogallo. Autor: AGS-CTFC

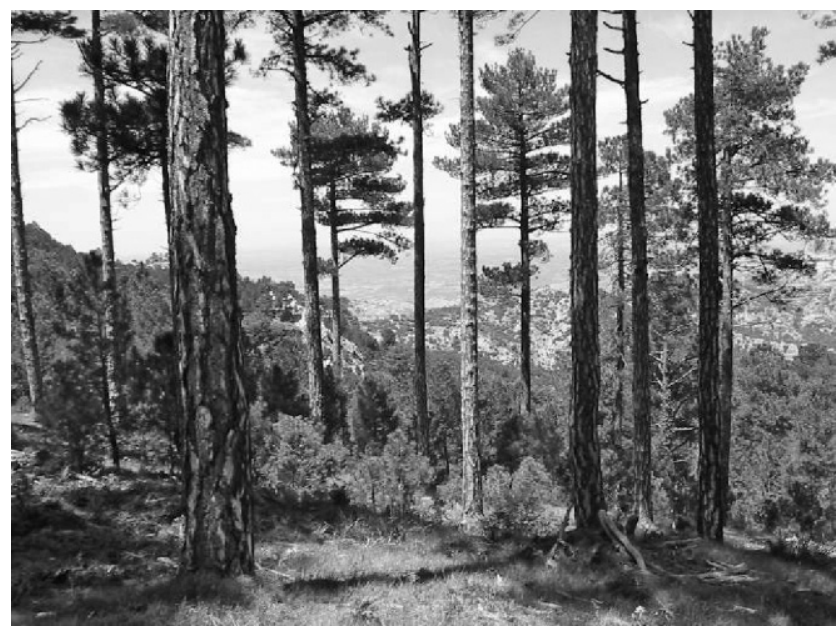

Figura 4. Parque Natural de Ports (Tarragona). Rodal de Pinus nigra maduro, resultado de la gestión realizada en el pasado en la zona. Autor: AGS-CTFC

\section{¿Por qué se declaran los ENP?}

Conocer el porqué se crea un ENP, es decir, las motivaciones y criterios que han sido determinantes para su denominación, es básico para fijar los objetivos preferentes que se buscan/esperan para ese espacio, y poder así planificar y gestionar de acuerdo a ellos.

Entre los principales motivos para la creación de un ENP y por tanto objetivos que se esperan conseguir con su declaración estarían (EUROPARC ESPAÑA, 2013):

- Conservación de la naturaleza: conservación de elementos naturales singulares, frágiles, amenazados o de especial interés ecológico, científico o paisajístico; conservación de hábitats concretos; o bien, conservación de la diversidad biológica en general, por su papel fundamental en el funcionamiento de 
los ecosistemas naturales y servicios que éstos proporcionan.

- Conservación de los servicios ambientales: producción de alimentos, agua o madera; regulación del ciclo del agua y de nutrientes; regulación de la fertilidad de los suelos y la protección de las cuencas hidrográficas o el control de la erosión.

- Conservación de los valores culturales y usos asociados a ese espacio.

Así pues, para asegurar realmente la conservación de un ENP, y planificar una gestión de acuerdo a los objetivos concretos por los que se creó y que compatibilice los usos que allí se den, es importante utilizar criterios técnicos-científicos que nos permitan fundamentar si los objetivos de conservación se están consiguiendo. Para ello, es básico hacer uso de indicadores de fácil estimación en campo que nos permitan monitorizar y controlar si un determinado valor a conservar (p. ej. elemento natural singular, hábitat, servicio ambiental, etc.), razón de ser de ese ENP, se está manteniendo, ha mejorado su estatus o, por lo contrario, está empeorando.

Por ejemplo, para el caso de la conservación de la diversidad biológica existen numerosos indicadores y criterios objetivos que valoran su estado, éstos incluyen: diversidad de especies, estructura y madureza del bosque, madera muerta, etc. (IUFRO, 2003; CAMPRODÓN, 2007; REQUE, 2008; VV.AA., 2009). Indicadores similares que nos pueden ayudar a determinar el estado de un ENP en relación a la conservación de servicios ambientales, como protección de suelos, cuencas, etc. también existen; así como criterios de ayuda a la diagnosis de la vulnerabilidad de un espacio frente al cambio global o los incendios, una de las principales amenazas de nuestros ecosistemas. Todos estos criterios deberían integrarse en la planificación y gestión de un ENP.

Por tanto, es importante evaluar si los ENP continúan cumpliendo de forma eficiente las funciones y objetivos por los que se crearon y cual es su estado de conservación, así como analizar las causas, si es el caso, que puedan motivar su vulnerabilidad o problemas de persistencia. No es extraño encontrar espacios forestales, que fruto de políticas conservacionistas, basadas en no intervenir o gestionar, presentan valores de biodiversidad o ambientales en peor estado que otros en los que se compatibiliza de manera sostenible la conservación con la producción de bienes y servicios.

La cuestión es, ¿qué debemos hacer para conservar los valores y usos de los ENP?

\section{CONSERVACIÓN DE LOS ENP: GESTIÓN VS. NO GESTIÓN}

Se conoce que la conservación de los valores naturales y de sus servicios ecosistémicos, así como los valores culturales asociados a un bosque, necesitan de una gestión forestal sostenible.

Sin embargo, algunos grupos sociales han percibido durante tiempo que algunas de las funciones que se esperan para los ENP, a simple vista, no requieren de la gestión, como por ejemplo, protección de la fauna y la biodiversidad. Cabe apuntar al respecto que la no-gestión es una opción de gestión siempre que se integre en un esquema planificado, de lo contrario es abandono. La disyuntiva gestión-no gestión ha sido ampliamente debatida en el pasado reciente y tiene su origen en la consideración de los sistemas forestales como sistemas naturales con dinámicas propias e independientes del hombre (GONZÁLEZ et al., 2011).

Lo cierto es que los sistemas forestales han evolucionado bajo influencia antrópica desde muy antiguo y tienen mucho de paisaje cultural, hasta el punto de que prácticamente nada de cuanto nos rodea es explicable de forma satisfactoria sin contar con el hombre y su acción secular, directa e indirectamente. Así pues, la mayoría de los ENP se corresponden con formaciones no sólo íntimamente ligadas a determinados usos humanos, sino incluso resultantes de los mismos (EZQUERRA, 2010), hecho generalizado en toda la cuenca mediterránea (NOCENTI \& COLL, 2013).

Aun así, por encima de los diferentes enfoques sociales y el conflicto de valoraciones expuesto, existe hoy consenso en que la conservación de los valores naturales y de sus servicios ecosistémicos, así como los valores culturales asociados a un bosque, necesitan de una gestión forestal sostenible, dicho de otra manera que la conservación de los ENP y su desarrollo en condiciones adecuadas requiere de una gestión activa y que su abandono no garantiza su conservación en la actualidad (GoNZÁLEZ et $a l$., 
2011), como seria el caso de espacios forestales abandonados que han acumulado mucha biomasa en los últimos decenios y que actualmente son muy vulnerables a los incendios forestales y efectos del cambio climático.

En definitiva, la gestión debe ser capaz de satisfacer los diferentes intereses y objetivos que convergen en un ENP de manera efectiva y eficiente, siempre teniendo muy presente el entorno socio-económico donde se encuentra el espacio protegido. La organización coherente de todos estos aspectos para alcanzar los objetivos perseguidos es compleja, y sólo es posible mediante una planificación táctico-operativa de calidad, a través de figuras de planificación como son los proyectos de ordenación forestal (POF) o planes de técnicos de gestión forestal (PTGMF).

\section{IMPORTANCIA DE UNA PLANIFICACIÓN ORGANIZADA DE LOS ENP}

\section{Disparidad de figuras}

Como ya se ha comentado, en España, existen más de 40 denominaciones para designar los ENP y una gran diversidad de normativas sectoriales y figuras de planificación a escala territorial que afectan a estos espacios, lo cual dificulta, a menudo, la implementación de figuras de planificación a nivel táctico y operativo y, en definitiva, la gestión final del ENP.

Por ejemplo, para el caso de Cataluña, ésta dispone de una variada red de espacios protegidos (GENCAT, 2013), donde las figuras de protección establecidas por la Ley $12 / 1985$, de Espacios Naturales, son diversas y, además, en su gestión intervienen diferentes administraciones. La Ley establece las siguientes categorías: parques nacionales, parajes naturales de interés nacional, reservas naturales y parques naturales.

En total, el sistema de áreas protegidas de Cataluña equivale una superficie del $30 \%$ del territorio y comprende: 1 parque nacional, 13 parques naturales, 4 parajes naturales de interés nacional y 1 reserva natural. De estos, 14 ENP están gestionados por el Departamento de Agricultura, Ganadería, Pesca, Alimentación y Medio Natural de la Generalitat de Catalunya y el resto por otras 5 entidades más.
Esto es una muestra de la disparidad de figuras y de responsabilidades sobre la gestión de los ENP, hecho que también se da en todo el territorio español, a parte, algunos ENP corresponden a montes públicos de ayuntamientos y otros, una gran mayoría, a fincas de titularidad privada, lo cual todavía hace más compleja la organización y planificación consensuada de estos espacios.

\section{Disparidad de planificación: necesidad de integrar}

Hay dos instrumentos legales para planificar los ENP: los planes de ordenación de los recursos naturales (PORN y equivalentes), normalmente de carácter estratégico e indefinido, y los planes rectores de uso y gestión (PRUG o equivalentes), a nivel táctico, donde se establecen en periodos de varios años las medidas de gestión a desarrollar. Según datos de EUROPARC ESPAÑA (2013), actualmente alrededor del $40 \%$ de la superficie declarada como Parque Nacional y el $90 \%$ de la superficie declarada como Parque Natural está incluida en un PORN y el $68 \%$ de parques nacionales, el 55\% de los parques naturales y el $30 \%$ de las reservas tienen vigente su correspondiente PRUG, sin embargo pocos gozan de POF o PTGMF.

A parte, cada comunidad autónoma tiene sus figuras de planificación como planes especiales, planes municipales, planes específicos, etc. En la Tabla 1 se muestra un ejemplo autonómico concreto (Cataluña) de estructura de planificación y normativa que afecta a los espacios forestales en general, incluidos los ENP, que muestra la complejidad de realizar una planificación operativa y la necesidad de integrar la planificación.

Así pues, podría darse el caso de que exista un Plan de Ordenación de los Recursos Forestales (PORF) que afecte a un ENP y que utilice criterios referentes a la conservación de la biodiversidad diferentes a los que contemplan otras figuras de planificación que afectan ese ENP. El hecho de que sobre un mismo territorio coexistan múltiples instrumentos tan similares en sus objetivos y tan diferentes en sus determinaciones no es recomendable, pues complica extremadamente la consecución de los objetivos concretos y reales que se quieran llevar a cabo en el ENP (EZQUERRA, 2010). 


\begin{tabular}{|c|c|c|c|c|}
\hline \multicolumn{5}{|c|}{ Ámbitos de planificación } \\
\hline $\begin{array}{l}\text { Escala } \\
\text { territorial }\end{array}$ & $\begin{array}{l}\text { Planificación y } \\
\text { Gestión forestal }\end{array}$ & \begin{tabular}{|l|} 
Prevención \\
incendios forestales
\end{tabular} & $\begin{array}{l}\text { Espacios Naturales } \\
\text { Protegidos }\end{array}$ & $\begin{array}{l}\text { Otras figuras de } \\
\text { Planificación }\end{array}$ \\
\hline Cataluña & $\begin{array}{l}\text { Plan General de } \\
\text { Política Forestal }\end{array}$ & - & $\begin{array}{l}\text { Plan de Espacios de } \\
\text { Interés Natural } \\
\text { Red Natura } 2000\end{array}$ & \begin{tabular}{|l|} 
Plan Territorial General de \\
Cataluña Planes Territoriales \\
Sectoriales (p. ej. energía \\
eólica, etc.) \\
\end{tabular} \\
\hline $\begin{array}{l}\text { Grandes } \\
\text { unidades } \\
\text { geográficas }\end{array}$ & \begin{tabular}{l|} 
Plan de \\
Producción \\
Forestal $^{1}$ PORF $^{2}$
\end{tabular} & - & $\begin{array}{l}\text { Plan de recuperación } \\
\text { especies en peligro (p. ej. } \\
\text { quebrantahuesos, nutria, etc.) }\end{array}$ & $\begin{array}{l}\text { Planes Territoriales } \\
\text { Parciales (p. ej. régimen } \\
\text { de protección del suelo, } \\
\text { paisaje, etc.) } \\
\end{array}$ \\
\hline $\begin{array}{l}\text { Macizos/masas } \\
\text { boscosas } \\
\text { continuas }\end{array}$ & - & \begin{tabular}{|l|} 
Perímetros de \\
Protección Prioritaria
\end{tabular} & - & $\begin{array}{l}\text { Zonas de Actuación Urgente } \\
\text { (ZAU) tras incendio forestal }\end{array}$ \\
\hline $\begin{array}{l}\text { Unidades } \\
\text { territoriales } \\
\text { de alto valor } \\
\text { natural }\end{array}$ & - & - & $\begin{array}{l}\text { PORN }^{3} \\
\text { PRUG }^{3} \\
\text { Plan Especial de protección } \\
\text { del medio natural y del } \\
\text { paisaje } \\
\text { Plan de gestión y directrices }^{4} \\
\text { de la Red Natura } 2000 \\
\end{array}$ & - \\
\hline Comarca & - & $\begin{array}{l}\text { Plan Comarcal de } \\
\text { Prevención }\end{array}$ & - & $\begin{array}{l}\text { Planes Directores } \\
\text { Territoriales } \\
\text { Planes Comarcales } \\
\text { de Montaña } \\
\end{array}$ \\
\hline Municipio & - & $\begin{array}{l}\text { Plan de Prevención } \\
\text { Municipal }\end{array}$ & $\begin{array}{l}\text { Plan Especial de protección } \\
\text { i gestión del patrimonio } \\
\text { natural municipal }\end{array}$ & - \\
\hline $\begin{array}{l}\text { Monte/ } \\
\text { propiedad } \\
\text { forestal }\end{array}$ & \multicolumn{4}{|c|}{ Proyecto de Ordenación Forestal (POF) / Planes Técnicos de Gestión y Mejora Forestal (PTGMF) } \\
\hline \multicolumn{5}{|c|}{$\begin{array}{l}\text { ' Contemplados en la Ley Forestal de Cataluña. No se ha elaborado ninguno hasta la fecha. } \\
{ }^{2} \text { Contemplados en la Ley de Montes del } 2003 \text { (Ley 43/2003, de } 21 \text { de noviembre). En fase de elaboración. } \\
{ }^{3} \text { A pesar de ser el PORN y PRUG figuras de planificación territorial previstas en la Ley Estatal 4/89, hay pocos ENP en Cataluña } \\
\text { que dispongan actualmente de estas planificaciones, la mayoría están en fase de elaboración. } \\
{ }^{4} \text { Figura que tiene su origen en los Planes Especiales previstos en las Leyes sobre Régimen del Suelo y Ordenación Urbana de } \\
1956 \text { y 1976. Esta figura queda recogida también en la normativa urbanística y de Espacios Naturales Protegidos autonómica } \\
\text { (equivalente al PORN). }\end{array}$} \\
\hline
\end{tabular}

Tabla 1. Ejemplo de figuras de planificación con afectación a los espacios forestales en Cataluña, incluidos los Espacios Naturales Protegidos (GONZÁLEZ et al. 2011)

Por ello, es imprescindible la coordinación entre los distintos instrumentos de planificación del territorio. Las figuras de planificación a escala monte deben incorporar la zonificación y directrices de las figuras de planificación de escala espacial y jerárquica superior. Del mismo modo, las figuras de planificación de ámbito superior necesitan adaptarse a la realidad y necesidades que ponen de manifiesto las planificaciones a escalas menores.

En este sentido, Europarc EsPaÑa (2008) expone la necesidad de la integración de la pla- nificación y la gestión activa de espacios naturales protegidos y de la coherencia en la planificación en escala de los ENP. Por tanto, no debe existir duplicidad de planificación sobre un mismo ENP y deben integrarse los diferentes documentos de planificación que puedan existir, de esta manera, por ejemplo, si existe un Proyecto de Ordenación Forestal (POF) de un monte incluido en una Zona de Especial Conservación (ZEC), el Plan de gestión de la ZEC debe integrar la planificación del POF y al revés, si una ZEC dispone de un Plan de gestión 
y se realiza un POF, éste debe integrar dicho plan (SCHWENDTNER et al., 2009)

Los POF son perfectamente válidos para planificar las gestión de ENP y zonas de la Red Natura 2000 desde la perspectiva de la Directiva Hábitats, para ello es importante incorporar medidas y criterios de conservación de la biodiversidad y servicios ambientales en la gestión silvícola. El nivel de detalle de los POM es mayor, por lo cual incluso es preferible abordar la gestión de las ZEC desde estos, para ello es imprescindible el entendimiento entre los distintos órganos administrativos que planifican e idealmente tener la planificación unificada en un solo documento (VERICAT y PIQUÉ, 2007; SCHWENDTNER et $a l .$, 2009).

En cualquier caso, es sumamente importante evitar la duplicidad y, más aun, el confrontamiento de las planificaciones, de manera que la gestión prescrita en los POM debe ser igual a la de los Planes de gestión de las ZEC.

Pero la realidad es que poca superficie comprendida dentro de los ENP dispone de figuras de planificación a escala operativa, en las que se articulen y concreten en el espacio y tiempo actuaciones de gestión con el fin de asegurar la conservación del ENP y su compatibilización con los diferentes funciones y usos que se dan en estos espacios.

En el caso de la Directiva Hábitats, ésta contempla la necesidad de realizar planes de gestión para los espacios de la red, para asegurar su conservación, aunque no establece la obligatoriedad de redactar planes de gestión a nivel operativo. La pregunta es si desde la Ordenación Forestal podemos dar respuesta a ese reto y la respuesta es que sí (Olabe y Val, 2007, Vericat y Piqué, 2007, SCHWENDTNER et $a l$., 2009), por tanto no es necesario buscar nuevos instrumentos de planificación de los ENP, LIC, ZEC, cuando ya disponemos de una herramienta que nos permite una planificación integral con el objetivo de preservar los valores ecológicos y compatibilizando los usos y aprovechamientos que se dan en la zona, desde la perceptiva de un gestión forestal sostenible.

Al final, cada Comunidad Autónoma en relación a la gestión de la Red Natura 2000 ha desarrollado sus propias directrices o instrucciones, como es el caso de Murcia (CARM, 2012) o Cataluña (GENCAT, 2006), entre otras. Ésta última, por ejemplo, contempla explícitamente en sus instrucciones la necesidad de elaborar, en el marco de la planificación de la Red Natura 2000, PO y PTGMF.

\section{RED NATURA 2000, UNA OPORTUNIDAD PARA LA GESTIÓN DE LOS ENP}

La Red Natura 2000 es el principal instrumento en materia de conservación de la naturaleza de la política europea. Se desarrolla a partir de la Directiva Aves (1979) y Directiva Hábitats (1992) en las que se definen el tipo de áreas, los criterios de designación y los procesos para el seguimiento y la gestión de las mismas. Es una Red donde se recogen los territorios europeos que contienen especies y hábitats de relevancia a escala europea, y está compuesta por los llamados Lugares de Importancia Comunitaria (LIC), hasta su transformación en Zonas Especiales de Conservación (ZEC) y las Zonas de Especial Protección para las Aves (ZEPA).

La Red Natura 2000 ocupa el 17,5\% de la Unión Europea, siendo España el país que aporta más superficie terrestre a la red, con más de $137.000 \mathrm{~km}^{2}$ (seguida de Francia con 68.000 $\mathrm{km}^{2}$ ), esto representa más del $27 \%$ de su superficie terrestre (MAGRAMA, 2013b).

En España, la Red está compuesta por 1.446 LIC y 595 ZEPA, estando, en muchos casos, las superficies de LIC y ZEPA superpuestas en el territorio. Están representadas cuatro de las nueve regiones biogeográficas definidas para el conjunto de Europa (Macaronésica, Mediterránea, Atlántica y Alpina). De éstas, la región biogeográfica mediterránea es la que aporta más superficie absoluta a la Red Natura 2000 en nuestro país, con casi 11,5 millones de hectáreas.

En el proceso de declaración de la red, cada comunidad autónoma ha propuesto el territorio a incluir en la red, en el caso de Cataluña, por ejemplo, se integran en la Red Natura 2000, los espacios que forman parte de la Red de Parajes de Especial Interés Nacional (PEIN), tanto públicos como privados. En Navarra, se proponen como ZEC los Montes Catalogados de Utilidad Pública que constituyen hábitats naturales de interés comunitario.

Así pues, la mayoría de los ENP coinciden con las áreas de la Red Natura 2000, en las que 
las prioridades de conservación deberían establecerse en función de lo indicado en la Directiva Hábitats.

En este sentido, como se comenta en el apartado anterior, la Red Natura 2000 puede ser una oportunidad para integrar la disparidad de planificaciones, al contar con un marco de planificación de nivel superior a todas ellas, asegurando una planificación integrada en las políticas territoriales y sectoriales y garantizando un sistema de planificación en cascada, en el que se aborde desde la planificación estratégica a la operativa, a través de la figura de POF vigente, integrando criterios de conservación de la biodiversidad en la selvicultura y gestión forestal. Gestionar la superficie del territorio español comprendida en los ENP y/o Red Natura 2000 es un reto y una oportunidad para los forestales (OLABE Y VAL, 2007).

De hecho, actualmente ya existen muchos ejemplos de gestión de espacios de la Red Natura 2000, como es el bosque de Valsaín en Segovia, zona de Alcudie en Navarra o Monte de Aralar en el País Vasco, entre otros (COMISIÓN EUROPEA, 2003), pero todavía es mucha la superficie protegida que requiere de un planificación y gestión para su conservación.

CANTERO (2007) presenta una relación de los diferentes tipos de Hábitats arbolados españoles, haciendo referencia a las principales características de su gestión actual y problemas asociados a la conservación de estos hábitats, la mayoría de ellos relativos al peligro de fuegos forestales, escasa regeneración y sobretodo en el caso de los Quercus, intenso aprovechamiento secular. Es importante integrar en la planificación de estos espacios todos estos aspectos de manera extremadamente urgente.

En definitiva, la Red Natura 2000 es una oportunidad para poner la selvicultura al servicio de conservación de los valores naturales.

\section{PLANIFICACIÓN DE LOS ENP Y CAMBIO GLOBAL}

\section{Características de los ENP: contexto de cambio global}

Como ya se ha comentado, en España casi 11,5 millones de hectáreas de la región biogeográfica mediterránea están presentes en la Red Natura
2000, eso da una idea de la gran representatividad del bosque mediterráneo dentro de los ENP.

Estos bosques presentan diferentes características en función de su situación y especies principales que los componen, pero en cualquier caso la mayoría de ellos se caracterizan por:

- Una gran diversidad biológica y riqueza de especies de flora y fauna.

- Una gran variedad de estaciones forestales, debido a la diversidad climática, edáfica y fisiográfica del territorio. Las estaciones forestales, en el contexto mediterráneo, condicionan extremadamente la composición específica de los montes a nivel de rodal, así como su estado vegetativo, crecimiento y riqueza biológica, entre otros. Por ello, deberán tenerse muy en cuenta en la toma decisiones sobre la gestión forestal.

- Una historia de usos intensa y diversa, al ser ecosistemas muy antropizados.

- Una gran multifuncionalidad, ofreciendo bienes y funciones como la producción de madera y productos forestales no madereros, biodiversidad, valor medioambiental, recreo, paisaje, etc.

- Una elevada vulnerabilidad a perturbaciones, principalmente incendios forestales, y a condiciones de estrés hídrico, que seguramente se agravarán en el actual contexto de cambio global.

En muchos casos, estos ENP constituyen bosques relativamente jóvenes, fruto de la colonización natural, de antiguos campos de cultivos y zonas de pasto, producida progresivamente desde mediados del siglo XX y repoblaciones realizadas. A la vez, a menudo las formaciones forestales presentan situaciones de elevada densidad, estancamiento del crecimiento e inestabilidad, por falta de gestión o gestión de baja calidad, lo que confiere estructuras forestales complejas de gestionar que necesitan de tratamientos selvícolas de mejora $\mathrm{y}$, además, muy vulnerables a los incendios forestales, por presentar gran acumulación de combustible y continuidad de vegetación tanto horizontal como vertical.

También existen ENP en zonas, preferentemente de montes públicos, que han sido gestionadas mediante proyectos de ordenación forestal, des de inicios del siglo XX y en las que no se da esta complejidad y disparidad de estructuras y 
que se encuentran en mejor estado en relación a su vitalidad, estabilidad, riqueza y vulnerabilidad a los posibles efectos del cambio climático.

Por otro lado, muchos bosques han estado intensamente explotadas desde antaño, como es el caso de los bosques de quercíneas, que ocupan en España más de 7 millones de has y muchos de ellos situados en ENP. Estas formaciones de Quercus mediterráneos han estado sometidas durante años al aprovechamiento de carbón y leñas y en algunas áreas se encuentran en un estadio avanzado de degradación y muy vulnerables a sequías, enfermedades y plagas y, sobretodo, incendios forestales (VERICAT et $a l .$, 2012).

Actualmente, muchos de los ENP ya se encuentran en una situación muy vulnerable en relación a los incendios forestales por su condición de ecosistemas mediterráneos, pero esta condición seguramente se agravará en el actual contexto de cambio climático, en el que se esperan periodos de sequía más largos y episodios climáticos extremos.

De todas las regiones bioclimáticas, la zona mediterránea está considerada una de las regiones más vulnerables al cambio global (EEA, 2008). Además de la componente climática, el área mediterránea presenta una serie de características socio-culturales que añaden una enorme complejidad a la hora de prever los posibles impactos del cambio global, al tratarse de sistemas forestales alterados desde hace milenios, e inmersos, en el caso de la cuenca norte occidental, donde se situaría España, desde hace décadas en una dinámica de cambio de usos del suelo que ha conlleva un incremento de superficie forestal y una notable acumulación de biomasa (FAO, 2011).

Entre las principales amenazas del cambio global destacan la reducción del crecimiento y de las producciones forestales, debilitamiento de las masas, decaimiento y mortalidad, problemas de regeneración, aumento de la frecuencia de incendios de mayor intensidad y tamaño, incremento de procesos erosivos y degradación del suelo, e incremento de problemas debido a plagas y enfermedades (REgato, 2008; PeÑUElas et al., 2010; SERRADA et al., 2011)

A la hora de prever los principales impactos negativos del cambio global sobre los ENP, debe considerarse su vulnerabilidad. Ésta depende tanto del carácter y magnitud del cambio como de la sensibilidad y capacidad de adaptación de las diferentes formaciones forestales presentes. Por tanto, los impactos tendrán diferente importancia en función de las especies concretas, área geográfica donde se localice el ENP, historia de su gestión y estado actual, principalmente.

\section{Gestión forestal y cambio global}

Las dos estrategias principales de respuesta al cambio global son la mitigación y la adaptación (IPCC, 2007). Numerosas prácticas de gestión forestal se alinean con estas dos estrategias. La mitigación trata de reducir la magnitud del cambio, actuando sobre las principales causas (por ejemplo, fijando más carbono atmosférico). Entre las prácticas de gestión forestal centradas en la mitigación, pueden citarse (IPCC, 2007c):

- Prácticas destinadas a incrementar la superficie forestal, como aforestaciones y reforestaciones, o densificación de masas abiertas, etc.

- Prácticas destinadas a incrementar la vitalidad y estabilidad de los bosques, incrementando su capacidad de fijar carbono, etc.

Por su parte, la adaptación trata de minimizar los impactos negativos del cambio sobre los sistemas forestales y sus funciones. Entre las prácticas de gestión forestal centradas en la adaptación se proponen:

- Fomento de la heterogeneidad espacial en especies y estructuras para incrementar la resistencia y resiliencia a incendios y plagas.

- Tratamientos de prevención de incendios, incluyendo uso del fuego prescrito y gestión de áreas estratégicas para incrementar la resistencia de los espacios forestales a los incendios.

- Utilización, en plantaciones, de mezclas de genotipos de varias zonas y distribuciones heterogéneas de pies para facilitar la transición a las futuras condiciones climáticas.

Por tanto, en la planificación de los ENP, la estrategia de promover la adaptación de las formaciones forestales al cambio global debe ser prioritaria. Para mejorar la capacidad de adaptación de un ENP, se pueden aplicar criterios selvícolas y de gestión forestal sostenible, como desbroces selectivos, clareos, claras, cortas de regeneración, etc., con el objetivo de incrementar su resistencia (reducir la exposición y la sensibilidad y, por lo tanto, la vulnerabilidad) y su resiliencia (absorber las perturbaciones manteniendo 
sus funciones), o favorecer una transición lo menos traumática posible a las nuevas condiciones mediante acciones de respuesta y reajuste.

En este sentido, es muy importante la gestión de los ENP con el objetivo de reducir la vulnerabilidad de las masas forestales frente a los grandes incendios, que propagan por las copas de los árboles con gran virulencia, y realizar una selvicultura orientada a promover formaciones forestales resistentes al paso del fuego y fomentar la capacidad de adaptación intrínseca al fuego que la mayoría de bosques mediterráneos tienen (PIQUÉ, 2012).

Por otro lado, como muchos científicos apuntan, las perturbaciones naturales tienen un importante rol en la conservación de la biodiversidad, sobretodo las de pequeña intensidad, a nivel de rodal forestal o bosque. De aquí, al ser el fuego un componente natural de la dinámica de las formaciones mediterráneas, reducir la acumulación de combustible de los ENP mediante fuegos de superficie de pequeña intensidad, intentando imitar un régimen de fuego natural, al igual que se hace en otras zonas como California, Australia o Sudáfrica, con las quemas controladas, puede ser una buena herramienta de naturalización de los ENP y gestión de la biodiversidad, y la vez evitar fuegos de alta intensidad que escapan de la capacidad de extinción actual (BROwN et al., 2004; AgEe \& SKINNER, 2005).

\section{PLANIFICACIÓN MULTIFUNCIONAL EN ENP}

La planificación multifuncional es clave para la conservación de los ENP, con el objetivo de preservar la biodiversidad y los recursos naturales, en el marco de una gestión forestal sostenible, que compatibilice la conservación con el aprovechamiento de los recursos. Además, la planificación debe tener muy presente la puesta en valor de los recursos naturales para poder hacer frente a los costes de gestión, e integrar las necesidades del territorio y medidas de adaptación al cambio global.

Es muy importante abordar la planificación de los ENP integrando la valorización del monte y las necesidades de la población local en lo que respecta al uso y aprovechamiento del espacio natural, de manera que el ENP les suponga una oportunidad de desarrollo y no una traba. Por ejemplo, generando actividades económicas ligadas a los valores y recursos naturales con que cuenta el ENP, siempre de manera sostenible. Por eso, es importante que el responsable de la planificación y el responsable de la gestión del ENP, conozcan la población del sitio, sus características socio-económicas y la relación que mantiene ésta con el ENP.

Así pues, la planificación forestal en ENP tiene como reto la multifuncionalidad, incluso más que en otros espacios forestales, puesto que muchas zonas consideradas como espacios protegidos son productivas y de propiedad privada, caso de Cataluña, por ejemplo, y en ellas debe realizarse un gran esfuerzo en compatibilizar la conservación de los espacios con el aprovechamiento forestal.

En definitiva, cabe apostar por una planificación y gestión multifuncional que integre los valores ecológicos, salud de los bosques y conservación de la biodiversidad, como objetivo preferente, y que a la vez asegure los beneficios sociales y económicos.

¿Como abordar esta planificación y gestión de los ENP, en el contexto actual de cambio global, que integre la multifuncionalidad, complejidad y biodiversidad de los bosques y los incendios forestales, como principal perturbación que afecta en el mediterráneo?

Es una tarea compleja, pero disponemos de herramientas y conocimiento para llevarla a cabo: por ejemplo, la gestión ecosistémica y adaptativa, teniendo en cuenta las dinámicas naturales que se observan y sin fijar esquemas fijos a largo plazo, o la gestión próxima a la naturaleza y de mínima inversión económica y, sobretodo, en cualquier caso, la gestión basada en una correcta diagnosis de las masas forestales y fijación de objetivos concretos para cada rodal, que nos permita una eficiente planificación multifuncional y asignación de los recursos.

\section{LA ORDENACIÓN FORESTAL COMO HERRAMIENTA DE PLANIFICACIÓN DE LOS ENP}

Existe consenso en que el Proyecto de Ordenación Forestal (POF) es una herramienta 
eficaz para la planificación integradora de los ENP (SECF, 2007).

Dehecho, los retos para la ordenación forestal del siglo XXI (Alí́A et al., 2011), contemplan, en gran medida, lo que una planificación de calidad de un ENP requeriría:

- Gestión ecosistémica y adaptativa, que considere las dinámicas de la vegetación y que rehúya de los esquemas a largo plazo.

- Integración real en la planificación de muchos otros aspectos: conservación de biodiversidad, calidad del paisaje, protección contra incendios, etc.

- Consideración de la multifuncionalidad, característica de los sistemas forestales de nuestro país, tanto en bienes como en servicios.

- Nueva consideración de los aspectos productivos, con una demanda de productos forestales distinta y renovada. Parece ser que la madera, tras décadas de bajos precios vuelve a tener interés económico, para su utilización como biomasa forestal primaria.

- Adaptación a una menor disponibilidad de recursos para la planificación a escala monte. Necesidad de figuras de planificación económicas y eficientes.

Es un hecho que la gran diversidad de normativas y disposiciones que confieren algún grado de protección a los ENP todavía hacen más compleja la planificación de los ENP, y es precisamente en éstos donde el carácter de la Ordenación Forestal como instrumento finalista de planificación integral de los terrenos forestales se hace más necesaria que nunca (GoNZÁLEZ et al., 2011):

- Por coherencia y eficiencia económica de la gestión.

- Para conseguir la coordinación de objetivos y actuaciones.

- De cara a la propiedad forestal, para abordar conjuntamente todos los aspectos que afectan a la gestión de su propiedad (ecológicos, productivos, medioambientales, etc.) en un solo documento de planificación.

El papel de instrumento único no es incompatible con la estructura administrativa, sino al contrario. La mayoría de las Comunidades Autónomas, y la propia Administración General del Estado, ubican bajo un mismo departamento la conservación de los espacios naturales y la gestión forestal. Paralelamente, en muchos casos los
Servicios de Fauna y Biodiversidad de la administración autonómica informan los instrumentos de ordenación forestal para su aprobación.

Si la ordenación forestal debe ser el instrumento unificado de planificación de la gestión forestal, ambiental y de conservación de la biodiversidad a escala monte, es necesario que sea capaz de integrar satisfactoriamente las directrices y estructuras territoriales de la normativa ambiental y los ENP, así como el resto de medidas de conservación.

En este sentido la Ordenación por Rodales, debido a su flexibilidad selvícola, espacial y temporal, es uno de los métodos de planificación más adecuado a la situación actual de muchos ENP. La ordenación por rodales da respuesta directa al requisito de flexibilidad y gestión adaptativa, pero además, ofrece al equipo redactor la posibilidad de aportar conocimientos y sentido común, innovar y lograr una planificación de calidad con la máxima eficiencia de recursos. El protagonismo en la planificación recae más que nunca en el equipo redactor, y no en el método de ordenación: la ordenación por rodales enfrenta al profesional forestal al reto y la responsabilidad de elaborar una planificación eficaz y eficiente.

\section{La ordenación por rodales y la conservación de la biodiversidad}

En una ordenación por rodales pueden coexistir una gran variedad de rodales de diferentes tamaños, con diferentes especies y mezclas, formas de masa y tipos de espacios abiertos. A un nivel superior, se adquiere una gran flexibilidad a nivel de paisaje, ya que el gestor tiene un grado elevado de libertad para combinar rodales de distinta cubierta forestal y modificar su forma y su distribución espacial, al menos a medio plazo.

Las ventajas genéricas de la Ordenación por Rodales en su utilización como herramienta de planificación de ENP serían (VERICAT Y PIQUÉ, 2007):

- Particularización del inventario a nivel de rodal, incorporando variables relacionadas con la biodiversidad si se considera necesario.

- Flexibilidad en la división dasocrática (libertad en la delimitación de los rodales), que permite una fácil incorporación de la zonificación propia del Espacio Natural Protegido. 
- Implementación de selviculturas diferenciadas a nivel de rodal, en función de los requerimientos de conservación. Fácil incorporación de las directrices de gestión propias del ENP y de otras medidas voluntarias de conservación y mejora de la biodiversidad, a escala de rodal y a escala de paisaje. En cuanto a la integración de criterios de conservación a escala rodal, pueden citarse: presencia de madera muerta, presencia de pies de grandes dimensiones, presencia de pies con oquedades, mantenimiento de la diversidad de especies arbóreas y ecotonos, grado de cobertura de matorral, etc.

A escala monte, la implementación de criterios de conservación se ve facilitada por la flexibilidad en la delimitación, forma, tamaño y localización de las unidades territoriales (rodales). Al mismo tiempo, el método posibilita la existencia de rodales con distintas formaciones vegetales (arboladas o desarboladas), con distinta composición específica, estructuras, edades, etc. Entre los criterios de conservación a nivel de monte pueden citarse: la diversidad de especies por rodales, gestión activa de áreas abiertas, conservación de rodales de bosque viejo, forma y tamaño de determinados tipos de rodal (conectividad, fragmentación, etc.), mantenimiento de estructuras en mosaico, etc.

La planificación de los usos a nivel de monte, la regulación de accesos, la red viaria, los usos ganaderos dispersos, las épocas de eje- cución de actuaciones planificadas, son también algunos de los aspectos a tener en cuenta.

A modo de ejemplo, en la Tabla 2 se resumen las principales medidas adoptadas en ordenaciones por rodales en espacios naturales de Pirineo y Prepirineo catalán, con el objetivo de integrar en la gestión criterios para la conservación de la biodiversidad (VERICAT Y PIQUÉ, 2007).

Áreas concretas de protección especial o áreas sensibles por la presencia de determinadas especies pueden estar formadas por uno o varios rodales y ser de extensión variable. La definición de objetivos y la gestión diferenciada a nivel de rodal permiten configurar una gestión óptima en áreas concretas de protección especial o áreas sensibles por la presencia de determinadas. Como ejemplo, en áreas especialmente sensibles de un monte incluido en un ENP se han seguido los siguientes pasos para definir la gestión (GoNZÁLEZ et $a l ., 2011$ ):

1. Identificar los sectores críticos para las especies o comunidades a conservar. Se entiende por sector crítico aquella zona del monte donde anualmente una especie desarrolla una parte vital de su ciclo biológico, que tiene importancia en el mantenimiento de la población. En un sentido amplio, puede incluir zonas de hábitat potencial, corredores, etc.

2. En los rodales incluidos en los sectores críticos, definir las directrices de gestión y objetivos para mejorar el hábitat en relación a las comunidades o especies de interés.

\begin{tabular}{|ll|}
\hline $\mathbf{1}$ & Selviculturas diferenciadas a nivel de rodal \\
\hline $\mathbf{2}$ & Medidas para mejorar la calidad del hábitat a escala de rodal \\
\hline & Árboles grandes y ramosos. Árboles con oquedades o nidos de grandes aves \\
& Árboles decrépitos, madera muerta y en descomposición \\
& Pies extracortables en cortas de regeneración en masas regulares \\
& Pies de gran diámetro en masas irregulares \\
& Diversidad de especies en el estrato arbóreo \\
& Cobertura adecuada de matorral \\
& Forma y estructura de la masa \\
\hline $\mathbf{3}$ & Medidas para mejorar la calidad del hábitat a escala de paisaje \\
\hline & Zonificación: distribución de usos \\
& Rodales de bosque viejo \\
& Rodales de no gestión o evolución natural \\
& Gestión activa de espacios abiertos \\
& Forma y distribución espacial de los rodales \\
\hline
\end{tabular}

Tabla 2. Grupos de medidas de gestión para la conservación de valores naturales utilizados en ordenaciones por rodales en el Pirineo y Prepirineo catalán (VERICAT Y PIQUÉ, 2007) 
3. Definir rodal a rodal la propuesta de gestión para que se cumplan los objetivos anteriores, incorporando los criterios de conservación a escala de rodal y monte que se consideren adecuados.

4. Adaptar la planificación de aspectos generales del monte (accesos, red de senderos, usos extensivos, etc.) a los objetivos de conservación propuestos para cada rodal, sector o monte.

Cuando estas áreas se encuentran dentro de un ENP, estos pasos se deben realizar en estrecha colaboración con los técnicos del ENP e integrando las directrices de gestión del mismo. Además, en todo caso, la planificación final de ENP debe realizarse en coordinación con el propietario del monte, público o privado, el gestor responsable de monte e integrar, en la mayor medida posible, las necesidades de la población local en lo que respeta al uso y aprovechamiento del ENP.

\section{BIBLIOGRAFÍA}

Agee, J.K. \& Skinner, C.N.; 2005. Basic principles of forest fuel reduction treatments. Forest Ecol. Manage. 211(1-2): 83-96.

Alía, R.; Bonet, F.J.; CoRTina, J.; DÍAz, F.; Gómez, A.; Navarro R.M.; Piqué, M.; RoJo, A.; RUÍZ, P.; RUÍZ, M.; VERICAT, P. \& ZAMORA, R.J.; 2011. Los bosques en España. En: L.M. Jiménez (ed.), Sostenibilidad en España 2011: 347-351. Mundi-Prensa. Madrid.

Brown, R.T.; AgEE, J.K. \& FrankLIN, J.F.; 2004. Forest restoration and fire: Principles in the context of place. Conservation Biology 18(4): 903-912.

CAmprodon, J.; 2007. Tratamientos forestales y conservación de la fauna vertebrada. En: J. Camprodon y E. Plana (eds.). Conservación de la biodiversidad y gestión forestal: su aplicación en la fauna vertebrada: 173-227. Edicions de la Universitat de Barcelona. Barcelona.

DudLeY, N. (ed); 2008. Directrices para la aplicación de las categorías de gestión de áreas protegidas. Gland, Suiza UICN. $\mathrm{x}+96 \mathrm{p}$

CANTERO, A.; 2007. La componente forestal en la Red Natura 2000 en España. Cuad. Soc. Esp. Cienc. For. 21: 101-112.
CARM; 2012. Gestión Integral de los espacios protegidos de la Red Natura 2000 del noroeste de la Región de Murcia. I, II. Consejería de Agricultura y Agua. Dirección General de Medio Ambiente. Comunidad Autónoma de la Región de Murcia. http://www.murcianatural.carm.es

COMISIÓN EUROPEA; 2003. Natura 2000 y los bosques: retos y oportunidades - Guía de interpretación. Oficina de Publicaciones Oficiales de las Comunidades Europeas, Luxemburgo.

EEA; 2008. Impacts of Europe's changing climate - 2008. An indicator-based assessment (EEA Report No 4/2008). Office for Official Publications of the European Communities, Luxembourg.

EUROPAC ESPAÑA; 2008. Planificar para gestionar los espacios naturales protegidos. Ed. Fundación Interuniversitaria Fernando González Bernáldez para los espacios naturales. Madrid.

EUROPAC ESPAÑA; 2013. Espacios Naturales Protegidos. http://www.redeuroparc.org

EzQUERRA, F.J.; 2010. Espacios protegidos y conservación: panorámica y principales retos. Los cambios de paradigma y la planificación integral. Cuad. Soc. Esp. Cienc. For. 31: 165-177.

FAO; 2011. State of Mediterranean Forests (SoMF). Concept paper. Roma.

GENCAT; 2006. Directrius per a la gestió dels espais de la Xarxa Natura 2000. http://www20.gencat.cat/

GENCAT; 2013. Xarxa d'Espais Protegits. Parcs Naturals de Catalunya. http://www20.gencat.cat/portal/site/parcsnaturals

GonZÁlez, J.M.; PIQUÉ, M. \& Vericat, P.; 2011. Manual de ordenación por rodales: gestión multifuncional de los espacios forestales. $2^{\mathrm{a}}$ ed. Organismo autónomo Parques Nacionales, Ministerio de Medio Ambiente y Medio Rural y Marino, Madrid.

IPCC; 2007. Climate Change 2007: Impacts, Adaptation and Vulnerability. Contribution of Working Group II to the Fourth Assessment Report of the Intergovernmental Panel on Climate Change. Cambridge University Press, Cambridge, UK.

IUFRO; 2003. Monitoring and Indicators of Forest Biodiversity in Europe: from ideas to 
operationality. Abstract Booklet. 12-15 nov, 2003, Florencia, Italia.

MAGRAMA; 2013a. Espacios Naturales Protegidos en España. http://www.magrama.gob.es

MAGRAMA; 2013b. Red Natura 2000 en España. http://www.magrama.gob.es

NocENTINI, S. \& COLL, LL.; 2013. Mediterranean forests: human use and complex adaptive Systems. In: C. Messier, K. Puetmann and D. Coates (eds), Managing Forests as Complex Adaptive Systems: Building resilience to the challenge of global change: 215-243. The Earthscan Forest Library.

Olabe, F. \& Val, Y.; 2007. La Red Natura 2000, una oportunidad para el sector forestal. Cuad. Soc. Esp. Cienc. For. 21: 113-118.

Peñuelas, J.; Gracia, C.; Filella, I.; Jump, A.; Carnicer, J.; Coll, M.; Lloret, F.; Curiel, J.; Estiarte, M.; Rutishauser, T.; Ogaya, R.; Llusià, J. \& SARDANS, J.; 2010. "Introducing the climate change effects on Mediterranean forest ecosystems: observation, experimentation, simulation, and management". Forêt Méditerranéenne, XXXI(4): 357-361.

PIQUÉ,M.; 2012. Reducción de la vulnerabilidad a los grandes incendios forestales (GIF). En: P. Vericat, M. Piqué \& R. Serrada (eds.), Gestión adaptativa al cambio global en masas de Quercus mediterráneos: 83-100. Centre Tecnològic Forestal de Catalunya. Solsona (Lleida).

Regato, P.; 2008. Adaptación al cambio global Los bosques mediterráneos. UICN Centro de Cooperación del Mediterráneo, Málaga, España.
REQue, J.A.; 2008. Selvicultura en espacios naturales protegidos. En: R. Serrada, G. Montero \& J.A. Reque, J.A. (Eds). Compendio de Selvicultura Aplicada: 1005-1035. INIA y Fundación Conde del Valle Salazar. Madrid.

Schwendtner, O.; Olabe, F.; Val, Y. \& Baeza, E., 2009. Incorporación Práctica de los Objetivos de la Red Natura 2000 a la Ordenación de Montes. El ejemplo de Navarra. Com. oral. $5^{\circ}$ Congreso Forestal Español. Ávila.

SECF; 2007. Conclusiones de la reunión conjunta del grupo de trabajo de ordenación de montes y del de Espacios Naturales Protegidos sobre la Planificación Forestal en la Red Natura 2000. Cuad. Soc. Esp. Cienc. For. 21: 173-174.

Serrada, R.; Aroca, M.J.; Roig, S.; Bravo, A. \& GómEZ, V.; 2011. Impactos, vulnerabilidad y adaptación al cambio climático en el sector forestal. Notas sobre gestión adaptativa de las masas forestales ante el cambio climático. Ministerio de Medio Ambiente y Medio Rural y Marino. Madrid.

Vericat, P. \& PiQué, M.; 2007. Utilización del método de ordenación por rodales para compatibilizar la gestión forestal y la conservación de la biodiversidad. Cuad. Soc. Esp. Cienc. For. 21: 125-133.

Vericat, P.; Piqué, M. \& Serrada, R. (eds.); 2012. Gestión adaptativa al cambio global en masas de Quercus Mediterráneos. CTFC. Solsona.

W.AA., 2009. Bases ecológicas preliminares para la conservación de los tipos de hábitat de interés comunitario en España. Madrid: Ministerio de Medio Ambiente, y Medio Rural y Marino. Madrid. 